\title{
Simple and effective bacterial-based intratumoral cancer immunotherapy
}

\author{
Christina S E Carroll, ${ }^{1}$ Erin R Andrew, ${ }^{1}$ Laeeq Malik, ${ }^{2,3}$ Kathryn F Elliott, ${ }^{4}$ \\ Moira Brennan, ${ }^{4}$ James Meyer, ${ }^{5}$ Alexander Hintze, ${ }^{6}$ Andrew A Almonte, ${ }^{1}$ \\ Cassandra Lappin, ${ }^{4}$ Philip MacPherson, ${ }^{4}$ Klaus-Martin Schulte, ${ }^{3}$ \\ Jane E Dahlstrom, ${ }^{3,7}$ Rohit Tamhane, ${ }^{8}$ Teresa Neeman, ${ }^{9}$ Elizabeth W Herbert, ${ }^{5}$ \\ Maurice Orange, ${ }^{6}$ Desmond Yip, ${ }^{2,3}$ Rachel Allavena, ${ }^{4}$ Aude M Fahrer (D) ${ }^{1}$
}

To cite: Carroll CSE, Andrew ER, Malik L, et al. Simple and effective bacterialbased intratumoral cancer immunotherapy. Journal for ImmunoTherapy of Cancer 2021;9:e002688. doi:10.1136/ jitc-2021-002688

- Additional supplemental material is published online only. To view, please visit the journal online (http://dx.doi.org/10. 1136/jitc-2021-002688).

CSEC, ERA, LM and KFE contributed equally. MO, DY, RA and AMF contributed equally.

Accepted 14 July 2021

Check for updates

(C) Author(s) (or their employer(s)) 2021. Re-use permitted under CC BY-NC. No commercial re-use. See rights and permissions. Published by BMJ.

For numbered affiliations see end of article.

Correspondence to

Dr Aude M Fahrer;

aude.fahrer@anu.edu.au

\section{ABSTRACT}

Background We describe intratumoral injection of a slow-release emulsion of killed mycobacteria (complete Freund's adjuvant (CFA)) in three preclinical species and in human cancer patients.

Methods Efficacy and safety were tested in mammary tumors in mice, in mastocytomas in mice and dogs, and in equine melanomas. In mice, survival, tumor growth, and tumor infiltration by six immune cell subsets (by flow cytometry) were investigated and analyzed using Cox proportional hazards, a random slopes model, and a full factorial model, respectively. Tumor growth and histology were investigated in dogs and horses, as well as survival and tumor immunohistochemistry in dogs. Tumor biopsies were taken from human cancer patients on day 5 (all patients) and day 28 (some patients) of treatment and analyzed by histology. CT scans are provided from one patient.

Results Significantly extended survival was observed in mouse P815 and 4T1 tumor models. Complete tumor regressions were observed in all three non-human species (6/186 (3\%) of mouse mastocytomas; $3 / 14$ (21\%) of canine mastocytomas and 2/11 (18\%) of equine melanomas). Evidence of systemic immune responses (regression of noninjected metastases) was also observed. Analysis of immune cells infiltrating mastocytoma tumors in mice showed that early neutrophil infiltration was predictive of treatment benefit. Analysis of the site of mastocytoma regression in dogs weeks or months after treatment demonstrated increased $B$ and $T$ cell infiltrates. Thus, activation of the innate immune system alone may be sufficient for regression of some injected tumors, followed by activation of the acquired immune system which can mediate regression of non-injected metastases. Finally, we report on the use of CFA in 12 human cancer patients. Treatment was well tolerated. CT scans showing tumor regression in a patient with latestage renal cancer are provided.

Conclusion Our data demonstrate that intratumoral injection of CFA has major antitumor effects in a proportion of treated animals and is safe for use in human cancer patients. Further trials in human cancer patients are therefore warranted. Our novel treatment provides a simple and inexpensive cancer immunotherapy, immediately applicable to a wide range of solid tumors, and is suitable to patients in developing countries and advanced care settings.

\section{INTRODUCTION}

Induction of a systemic anti-cancer immune response by intratumoral injection of bacterial products has been previously proposed by our group ${ }^{1}$ and has been recently confirmed in a seminal paper. ${ }^{2}$ The rationale is that the immune system has evolved to recognize conserved pathogen-associated molecular patterns (PAMPs). Such recognition is the key to initiating a systemic immune response. Thus, injection of PAMPs directly into tumors has the potential to induce or restore the host immune response against the cancer.

Enlisting bacteria to treat cancers has a long history, dating back well over a century. Best known, historically, is the successful work of William Coley who repeatedly injected (every 2 to 3 days for several months) preparations of dead bacteria into hundreds of patients with inoperable cancers. For the first several years, he injected these 'Coley's toxins' into and around tumors. He later switched to intravenous injections. While both approaches resulted in durable cancer remissions in many patients (eg, of 312 inoperable sarcoma cases, 190 patients experienced complete tumor regressions ${ }^{3}$ ), it appears that he ultimately considered intratumoral injections most effective. ${ }^{4}$

More recently, interest has been revived in using bacteria and viruses to treat cancer. ${ }^{56}$ Several therapies are based on live, often 'oncolytic' bacteria or viruses. ${ }^{78}$ Others are based on dead bacteria or bacterial products. ${ }^{29}$ Regardless of the published rationale, in all these cases, modulation of the immune response is a likely mechanism of action. ${ }^{110}$

Unexpectedly, bacteria have also been implicated in the mechanism of action of chemotherapies $^{11-13}$ and of checkpoint inhibitor cancer therapies. ${ }^{14-16}$ We have recently proposed possible mechanisms for the latter. $^{17}$ 
It has been known for several years that bacteria can colonize intestinal tumors. ${ }^{18}{ }^{19}$ Very recently, bacteria have been identified in human cancers previously assumed to be sterile, ${ }^{20}$ and bacterial peptides have been identified on the major histocompatibility complex (MHC) molecules of melanomas, ${ }^{21}$ thereby providing an extra target for lysis of melanomas by bacteria-specific cytotoxic $\mathrm{T}$ cells. Thus, harnessing bacteria in anticancer therapies is a promising and rapidly evolving field of research.

Here, we describe in vivo trials of a novel form of intratumoral immunotherapy, injection of emulsified complete Freund's adjuvant (CFA). CFA is an extremely potent immune stimulant, developed in the $1950 \mathrm{~s} .{ }^{22}$ It is made up of three components: mineral oil and surfactant (together making up incomplete Freund's adjuvant (IFA), or Montanide ISA-51, which is already licensed for use in humans) and heat-killed Mycobacteria. Thus, both chemical components of CFA have previously been licensed for human use, and dead Mycobacteria are inherently safer than the live Mycobacteria used both in the widely administered childhood intradermal BCG vaccine against tuberculosis and as an intravesical treatment for superficial bladder cancer. If correctly emulsified, CFA forms a slow-release depot at the injection site, meaning that one injection provides continuous immunostimulation over a period of weeks. CFA therefore has the potential to be a simple and inexpensive form of cancer immunotherapy, accessible for use in both developed and emerging economies.

In this study, we describe the use of CFA in three preclinical species and in human cancer patients. We show that intratumoral injection of CFA induces anticancer responses in a significant proportion of treated animals, and is safe to use in human cancer patients.

\section{MATERIALS AND METHODS Complete freund's adjuvant}

CFA for mouse, dog, and horse experiments, and for the Canberra patient trial, was from Becton Dickinson (New Jersey, USA, catalog number: 263810) containing $0.5 \mathrm{mg} /$ $\mathrm{mL}$ Mycobacterium butyricum. CFA was emulsified 1:1 with sterile saline using two $5 \mathrm{~mL}$ Luer lock glass syringes or plastic syringes connected via an $18 \mathrm{G}$ microemulsifying needle (Cadence Science, Virginia, USA) to obtain a waterin-oil emulsion.

CFA used in Swiss patients 1 and 2 was from Sigma (Michigan, USA, catalog number: F5881). CFA used in patients 3 and 4 was AdjuLite from Pacific Immunology Corp (California, USA, catalog number: A5001). Both contain 1 $\mathrm{mg} / \mathrm{mL}$ Mycobacterium tuberculosis strain H37Ra. CFA used in the Swiss patients was emusified with Abnova Viscum (Pforzheim, Germany) or other non-fermented extracts of Viscum Album (Hiscia, Arlesheim, Switzerland).

\section{Mice and cell lines}

P815 (ATCC TIB-64) mastocytoma cells, 4T1 (ATCC CRL-2539) mammary adenocarcinoma and CT26.wt
(ATCC CRL-2638) colorectal carcinoma (American Tissue Culture Collection, Virginia, USA) were cultured in medium supplemented with $10 \%$ fetal calf serum (FCS), 1 $\mathrm{mM}$ sodium pyruvate, $0.1 \mathrm{M}$ HEPES, $100 \mathrm{IU} / \mathrm{mL}$ penicillin, and $100 \mu \mathrm{g} / \mathrm{mL}$ streptomycin (Gibco). P815 cells were cultured in Dulbecco's Modified Eagle Medium (DMEM) containing $4.5 \mathrm{~g} / \mathrm{L}$ D-glucose, and Glutamax (Gibco), 4T1 and CT26 cells in RPMI-1640 containing Glutamax (Gibco). Adherent cells were recovered using $0.25 \%$ trypsin (Gibco), and counted by hemocytometer, using Trypan Blue (SigmaAldrich) to exclude dead cells. Cells were re-suspended in 50\% medium without supplements, $50 \%$ phosphatebuffered saline prior to injection.

Prior to P815 cell injection, female DBA/2 J mice between 6 and 12 weeks of age (Animal Resource Centre, Perth, Australia) were shaved at the injection site and anesthetized ( $2 \%$ isoflurane). Tumors were then induced by subcutaneous (orthotopic) injection of $3 \times 10^{5} \mathrm{P} 815$ cells in a volume of $0.1 \mathrm{~mL}$. $4 \mathrm{~T} 1$ cells $\left(1 \times 10^{5}\right.$ cells in $\left.15 \mu \mathrm{l}\right)$ were injected orthotopically into the mammary fat pad or CT26 cells $\left(5 \times 10^{5}\right.$ in $\left.0.1 \mathrm{~mL}\right)$ were injected subcutaneously into the shaved injection site (non-orthotopic) of anesthetized female 6-week-old to 10-week-old Balb/c mice (bred at the ANU).

Tumors typically reached 4-7 $\mathrm{mm}$ (average of two perpendicular measurements measured using electronic calipers) 7-14 days after injection. As tumor size was slightly variable, prior to treatment, mice were divided into groups based on tumor size (to the nearest $0.5 \mathrm{~mm}$ ) and then mice in each size group were randomly assigned as treatment (0.05 mL intratumoral CFA) or control animals $(0.05 \mathrm{~mL}$ intratumoral saline), to ensure groups had similar numbers of mice of each tumor size. Each cage contained both treatment and control animals to avoid cage-to-cage variation. For many experiments, treatment was semiblinded, recorded separately (not on the cages), and sealed until the completion of the experiment, so as to prevent bias in tumor measurements. Tumors were measured using digital calipers every 1-2 days. Mice were euthanized when tumors reached $15 \mathrm{~mm}$ in diameter or showed signs of illness (hunched posture, inactivity).

\section{Analysis of tumor-infiltrating cells}

Fine-needle aspirates (FNAs) were taken with a 25G needle from anesthetized P815 tumor-bearing mice on days 1, 3, $5,8,11,14$, and 16 post-treatment, with individual mice receiving between two and five FNAs. ${ }^{23}$ Briefly, aspirates were digested at $37^{\circ} \mathrm{C}$ for $20 \mathrm{~min}$ using $5 \mathrm{mg} / \mathrm{mL}$ Collagenase type I (Worthington Biochemical, UK), $1.5 \mathrm{mg} / \mathrm{mL}$ Hyaluronidase (Sigma-Aldrich), and $0.01 \mathrm{mg} / \mathrm{mL}$ DNAse I (Worthington Biochemical) in DMEM supplemented with $1 \%$ FCS. Samples were then stained and analyzed by flow cytometry (LSRII, Becton Dickinson, USA; FlowJo, Treestar, USA). Two stains were used to identify immune cell types. Both stains contained a P815 tumor marker anti-CD63 (NVG-2) PE, pan-immune cell marker anti-CD45.2 (104) Alexa Fluor 488, and 7-aminoactinomycin-D (Life Technologies) for live/dead cell discrimination, as well as: Stain 
A-anti-CD8 $\alpha$ (53.6.7) APC Cy7 (BD Biosciences), antiCD4 (GK1.5) PE-Cy7, anti-CD25 (PC61) Brilliant Violet 421, anti-CD19 (6D5) Brilliant Violet 510, and anti-Pan-NK (CD49b) (Dx5) APC. Stain B-anti-CD11b (M1/70) Brilliant Violet 510, anti-CD11c (N418) PE Cy7, anti-F4/80 (BM8) Brilliant Violet 421, anti-Ly6G/Ly6C (GR1) (RB68C5) APC Cy7, and anti-Siglec-F (E50-2440) Alexa Fluor 647 (BD Biosciences, USA). All antibodies were from Biolegend (California, USA) unless otherwise specified.

\section{Dogs}

Dog experiments were approved by the University of Queensland institutional animal ethics committee (AEC approval numbers SVS/45/12; SVS/328/14/JMKT; ANRFA/SVS/039/14). Dogs naturally suffering from mastocytoma (mast cell tumor), previously diagnosed by either biopsy or cytologic examination by their referring veterinarian or a commercial diagnostic company, were recruited after owner briefing and completion of consent forms. Medical records, pathology reports, and recuts of paraffin-embedded sections from pretrial biopsies were obtained from referring veterinarians and commercial diagnostic companies with owners' consent.

Dogs underwent a full clinical examination by a registered veterinary surgeon with hematology and clinical chemistry profiles taken if required, to ascertain their clinical status and ability to tolerate anesthesia and treatment. At least 30 min prior to CFA treatment, fine-needle aspiration or surgical biopsy, dogs were premedicated with 1-2 $\mathrm{mL}$ of Histamil (chlorpheniramine maleate $10 \mathrm{mg} / \mathrm{mL}$; Ilium Troy laboratories Pty, Glendenning, NSW) to reduce the risk of mast cell degranulation anaphylactoid reactions caused by tumor handling. For biopsies, animals were anesthetized. Sedation was with acepromazine $(0.05 \mathrm{mg} / \mathrm{mL})$ and preoperative and postoperative pain relief was with methadone $(0.5 \mathrm{mg} / \mathrm{kg})$. Induction of anesthesia was with Alfaxan $(10 \mathrm{mg} / \mathrm{mL}$ alfaxalone; Jurox Pty, Rutherford, NSW) and maintenance with gaseous anesthesia, isoflurane (100\%).

Pretreatment incisional biopsy $(8 \mathrm{~mm}$ punch or wedge as clinically indicated) or FNA sample was taken where confirmatory diagnosis from a pathologist had not been previously achieved. Tumor biopsies were fixed in $10 \%$ neutral buffered formalin before routine paraffin embedding and staining with either H\&E or toluidine blue stain by routine methods. For lymphocyte identification, de-paraffinized formalin-fixed tumor sections were stained by routine immunohistochemical methods using Dako, clone F7.2.38 (Dako, Hamburg, Germany) for $\mathrm{CD}^{+} \mathrm{T}$ cells at 1:600 and AbD Serotec clone HM57 (Serotec, Oxford, UK) at 1:1800 for CD79a B cells as primary antibodies. Normal canine lymph node was used as a positive control and omission of primary was used as a negative control for each run. Canine mastocytomas were graded based on the schemes proposed by Kiipel et al..$^{24}$

Clinical assessments were performed at 1, 3, 6, and 12 weeks post-treatment as owner schedules allowed or on an ad hoc basis at owners' requests to address concerns or evaluate changes in clinical status. Dog well-being was further monitored with a questionnaire at 0 (pretreatment), $1,3,6,12$ weeks post-treatment, and as an ad hoc basis at later time points (The University of Queensland Institutional Human Research Ethics Approval 2014001294).

\section{Horses}

Gray horses diagnosed with melanoma were treated at the Adelaide Plains Equine Clinic (Gawler, South Australia). Informed consent was obtained from each owner and the veterinarian (author EWH) had general ethical research approval from the Department of Primary Industries and Regions, South Australia. Progression was followed by ultrasound measurements.

Equine melanoma biopsy material was fixed in $10 \%$ neutral buffered formalin before paraffin embedding, sectioning at 4 microns, and staining with either H\&E by routine methods. Serial sections of tumors were subjected to melanin bleach.

\section{Patients}

Swiss patients were treated at the Ita Wegman Klinik and Lukas Klinik, now merged into Klinik Arlesheim, Arlesheim, Switzerland. Patients volunteering for treatment signed informed consent forms, as required under Swiss law.

Australian trial: Australian New Zealand Clinical Trials Registry: ACTRN12616001637437. Due to administrative oversight, the trial was (retrospectively) registered after recruitment of the first patient, but before any further patients were recruited. The trial was approved by the ACT Health Human Research Ethics Committee (ETH.11.15.234) and by the Australian National University Human Research Ethics Committee (Protocol 2016/395), and all patients volunteering for treatment signed informed consent forms. In this ongoing doseescalation study, two cohorts of patients with advanced cancer (squamous cell carcinoma of the skin, clear cell renal cell carcinoma, adenocarcinoma of the prostate, transitional cell carcinoma of bladder, and cervical carcinoma) after exhausting standard of care therapies were treated with either $0.5 \mathrm{~mL}$ or $1 \mathrm{~mL}$ of CFA. CFA was administered into primary or metastatic cancer site either in clinic or under imaging guidance depending on tumor location. All patients underwent tumor biopsy 4 days after CFA administration. Clinical examination and laboratory assessments were performed every 6 weeks and CT scan repeated every 12 weeks. Patient history of BCG vaccination was not available. Tuberculin skin testing (TST) was not performed.

\section{Histology}

Four micrometer thick sections were made from formalinfixed paraffin-embedded tissue. Sections were stained with H\&E (Sigma-Aldrich, St Louis, Missouri, USA), and all specimens were examined by an experienced pathologist (JED). 

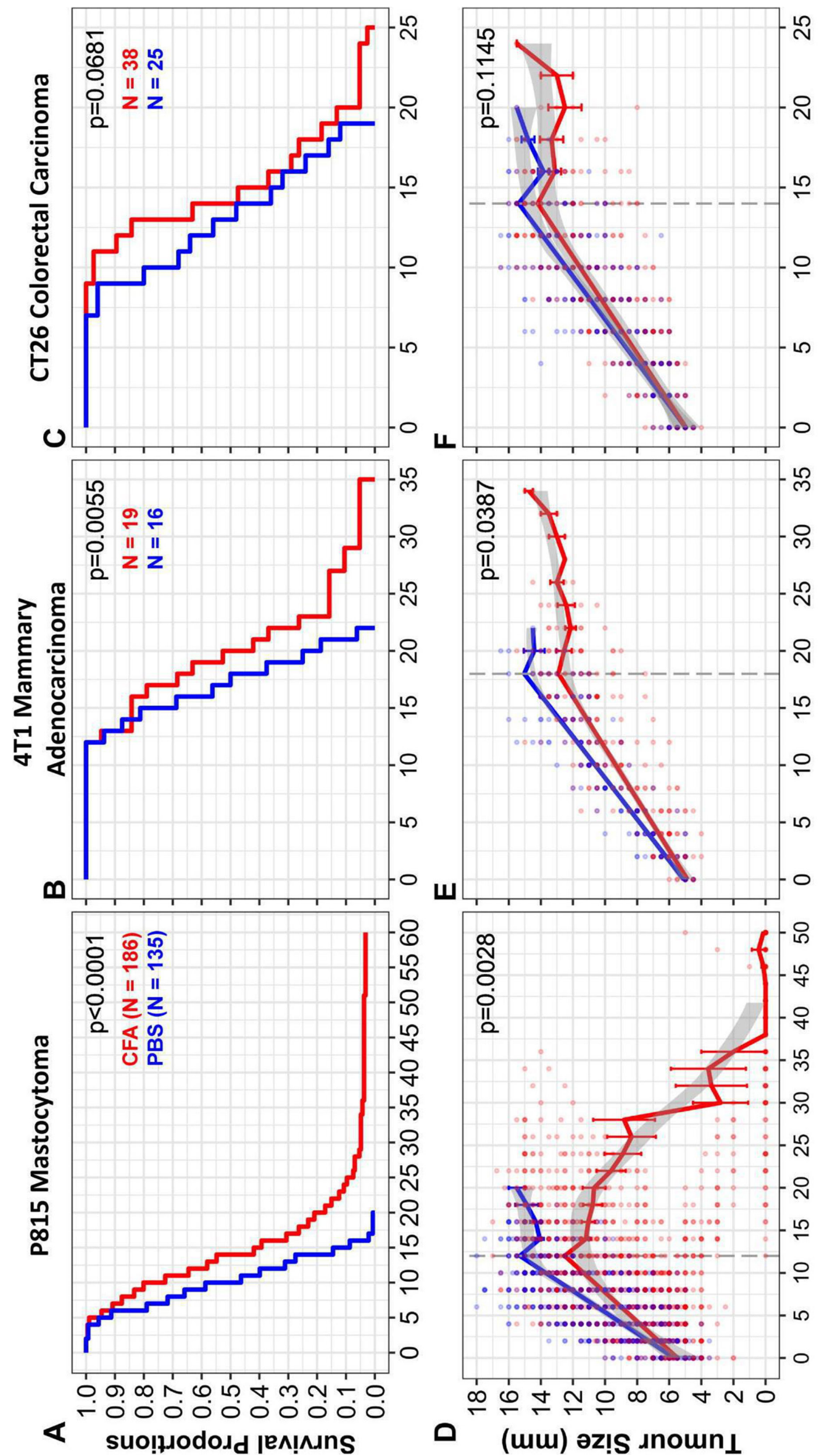

Figure 1 (Continued)

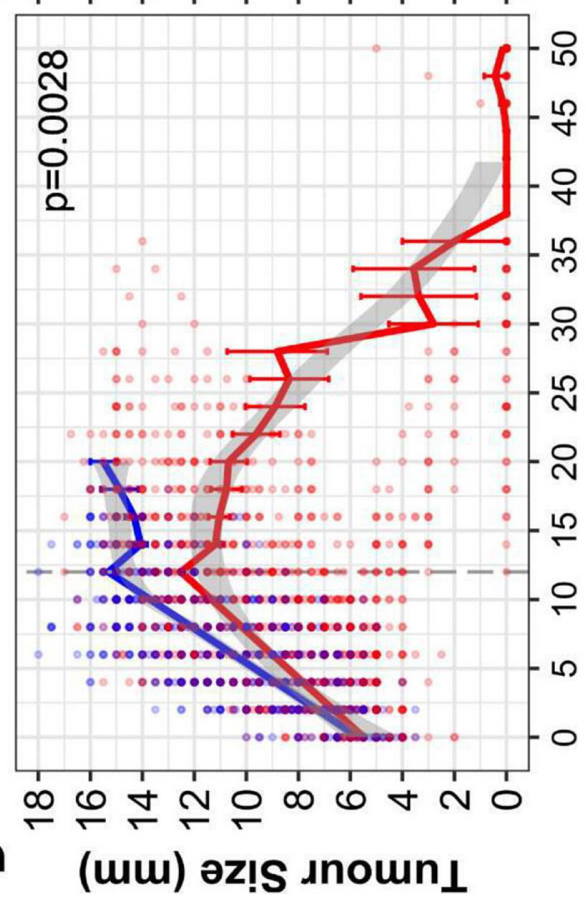


Figure 1 Treatment of solid tumors in mice with intratumoral CFA. Solid tumors were induced in 8-week-old to 12-week-old DBA/2J (P815) or BALB/c (4T1 or CT26) mice by subcutaneous injection of $3 \times 10^{5}$ (P815) or $5 \times 10^{5}$ (CT26) cells into the animals' flank or $1 \times 10^{5}$ cells into the mammary fat pad (4T1). Tumors were measured several times per week with digital calipers and the tumor size $(\mathrm{mm})$ was determined as the average of two perpendicular measurements. Mice were treated with intratumoral CFA (red) or phosphate-buffered saline (PBS) (blue) when tumors reached 4-7 mm in average diameter. (A-C) Survival curves of CFAtreated and PBS-control mice were analyzed using a Cox proportional hazards model. (A) CFA increases survival in P815 tumorbearing mice. CFA-treated mice had a statistically significant increase in survival compared with PBS-control mice $(p<0.0001$, HR: CFA:PBS=0.34, $95 \% \mathrm{Cl}(0.257$ to 0.438$)$ ). The median survival of CFA-treated mice was higher (14 days post-treatment) than PBS-control mice ( 10 days post-treatment). CFA $n=186$, PBS $n=135$. Data pooled from 31 separate experiments. Six complete tumor regressions were observed in CFA-treated mice. (B) CFA increases survival in 4T1 tumor-bearing mice. A statistically significant increase in survival was observed in CFA-treated mice compared with PBS controls $(p=0.0055, H R$ : CFA:PBS $=0.32,95 \% \mathrm{Cl}(0.145$ to 0.717$))$. The median survival of CFA-treated mice was 20 days compared with 17.5 days for PBS controls. CFA $n=19$, PBS $n=16$. Data pooled from three separate experiments. (C) CFA does not significantly increase survival in CT26 tumor-bearing mice. Statistical analysis did not show a significant effect of intratumoral CFA treatment on survival compared with PBS-control mice ( $p=0.0681$, HR: CFA:PBS $=0.61,95 \% \mathrm{Cl}(0.360$ to 1.037$))$. The median survival of CFAtreated mice was 14 days compared with 13 days for PBS controls. CFA $n=38$, PBS $n=25$. Data pooled from three separate experiments. (D-F) Tumor growth rates. To determine if the rate of growth differed between CFA-treated (red) and PBS-control treated (blue) mice, a random slopes model was developed using $\mathrm{R}$ software to analyze the interaction between treatment and tumor growth over time. Tumor growth slopes were compared up to the point at which approximately $50 \%$ of mice were euthanized (as the attrition of mice confounds the analysis beyond this point: P815=day 12, 4T1=day 18, CT26=day 14; represented by the dashed line), after which the raw means are shown. Error bars represent the SE. Tumor sizes for individual mice are shown by dots, with opacity set to $50 \%$ to visualize overlapping points. The gray shaded area indicates $95 \% \mathrm{Cl}$, determined by the locally estimated scatterplot smoothing (LOESS) method. (D) CFA decreases growth of P815 tumors. ANOVA $p=0.0028$, CFA $n=32$, PBS $n=29$. Data pooled from five separate experiments. (E) CFA reduces growth of $4 T 1$ tumors. ANOVA $p=0.148$, CFA $n=19$, PBS $n=16$. Data pooled from three separate experiments. (F) CFA does not reduce tumor growth of CT26 tumors. ANOVA $p=0.1145, C F A n=38$, PBS $n=25$. Data pooled from three separate experiments. Mice that received fine-needle aspirates were included in part A but not in parts B-F. ANOVA, analysis of variance; CFA, complete Freund's adjuvant.

\section{RESULTS}

\section{Mouse studies}

Efficacy of intratumoral CFA injection was tested in three mouse models of cancer: P815 mastocytoma, 4T1 metastatic breast cancer, and CT26 colorectal cancer. Efficacy was observed in P815 and 4T1 (figure 1A,B). In both cases, tumor cell lines were injected orthotopically (subcutaneously or in the mammary fat pad, respectively). Statistically significant efficacy was not observed in the non-orthotopically injected CT26 colorectal cancer model (figure 1C). The tumors were allowed to grow to an average of $5 \mathrm{~mm}$ in diameter. They were then injected once with $0.05 \mathrm{~mL}$ of CFA emulsified in saline or with saline alone as a control. Mice were euthanized when they became ill or if the cancer reached $15 \mathrm{~mm}$ in diameter. As shown in figure 1A,B, mice treated with CFA survived longer than control mice $(\mathrm{p}<0.00001$ for $\mathrm{P} 815, \mathrm{p}=0.0055$ for 4T1). Tumor growth was significantly slowed in the P815 model and 4T1 models (figure 1D,E) but not in the CT26 model (figure 1F). Illness is almost always due to metastatic burden, and CFA was shown to slow the progression of liver metastases in the P815 model (online supplemental figure 1A). Six mice treated for mastocytoma showed complete regression of their tumor (figure 1A and online supplemental figure 1B) and survived for up to 2 years without tumor recurrence.

IFA was injected intratumorally as an extra control in some experiments. It was found that IFA was no better than saline, thus the mycobacteria rather than the mineral oil emulsion is key to success of the treatment (online supplemental figure 1C).

\section{Canine studies}

Given our success in treating mastocytoma in the mouse model, and the high incidence and associated morbidity and mortality of mastocytoma in the pet dog population, a pilot clinical trial using intratumoral CFA was conducted on pet dogs suffering from spontaneous mastocytoma. Dogs suffering from spontaneous cancers are increasingly being recognized as an excellent comparative oncology model in which to study cancer therapies. ${ }^{25-27}$ Unlike mouse models seeded with rapidly dividing cell lines already selected for immune escape, canine cancers, like human cancers, will have developed over a long time frame and in the presence of the host immune response. Mastocytoma is common in dogs accounting for $7 \%-21 \%$ of canine cutaneous neoplasms. ${ }^{24}$ High-grade mastocytoma has a median survival time of less than 4 months and is associated with paraneoplastic syndromes causing high morbidity. ${ }^{24}$

As shown in table 1 , of 14 dogs treated with one injection of $0.05-0.2 \mathrm{~mL}$ of emulsified CFA, 3 dogs showed complete regression of their tumor, in 2 cases leading to substantially extended survival. The first in a Rottweiler whose nasal/lip mass returned following cessation of chemotherapy (predicted survival 8 weeks, actual 12 months), the second in a German short-haired pointer whose leg tumor recurred despite radical surgical resection (predicted survival 8-12 weeks, actual 16 months). Histology of pretreatment and post-treatment biopsies is shown in figure 2A, with few immune cells seen in the tumors before treatment, but residual immune cell infiltrates present following complete tumor regression. In 
the German short-haired pointer, another unsampled secondary mass previously diagnosed as a mast cell tumor regressed in addition to the injected tumor. Figure 2B shows immunohistochemistry on the injected tumor mass and on a de novo high-grade mastocytoma arising in the neck of the Rottweiler 17 weeks after treatment of his nasal/lip mass. B and T cells can be seen to clearly increase at the site of tumor regression and are also seen in a de novo neck tumor (not injected with CFA), which notably did not recur despite incomplete surgical regression. Further, the treatment was well tolerated (table 1). A subset of dogs which previously had ulceration of skin or mucous membrane prior to treatment exhibited some ulceration after CFA injection. In the Rottweiler on the days preceding lip mass regression, there was a 24-hour period of malaise and reduced activity reported by the owner. Thus, the side effect profile was favorable and could be classified as grade 1 on the veterinary cooperative oncology group-common terminology criteria for adverse events. ${ }^{28}$

These results show that treatment with CFA can result in complete regression of naturally occurring tumors and importantly, suggest that the treatment can have a systemic immune-mediated effect.

\section{Equine studies}

As well as treating dogs, the treatment was trialed in horses suffering from naturally occurring melanoma. Melanoma is a common problem in gray horses (affecting $>70 \%$ ), as the mutation causing the gray colouration also predisposes to melanoma. ${ }^{29}$ Tumors often occur around the tail, peri-anal area, lips and eyelids. ${ }^{30}$ While the disease progression is not as aggressive as human melanoma, it is a substantial cause of morbidity and mortality in gray horses due to space occupying effects in the perineum and throat and the presence of tumors within the viscera and thoracic organs. ${ }^{30}$ Few treatment options are available other than surgical resection, which is often impractical as the disease is multicentric.

Eleven horses were treated with intratumoral injections of $0.1-0.3 \mathrm{~mL}$ of CFA emulsified with saline into their melanomas. Details are given in online supplemental table 1. Representative histology is shown in online supplemental figure 2. Tumors were imaged by ultrasonography to follow progression of tumors after treatment. Three horses $(27 \%)$ showed evidence of clinical response. This included mass remissions and reductions in mass size.

\section{Correlation of immune infiltrate with survival}

To investigate the timing of immune infiltration into the tumor following CFA injection, we again made use of the mouse mastocytoma model. We have previously published a novel technique that allows serial quantitation of immune infiltrates without altering survival in mice injected with P815 tumors. ${ }^{23}$ As can be seen in figure 3, CFA treatment induced extensive infiltration of immune cells into the tumor compared with injection 
A

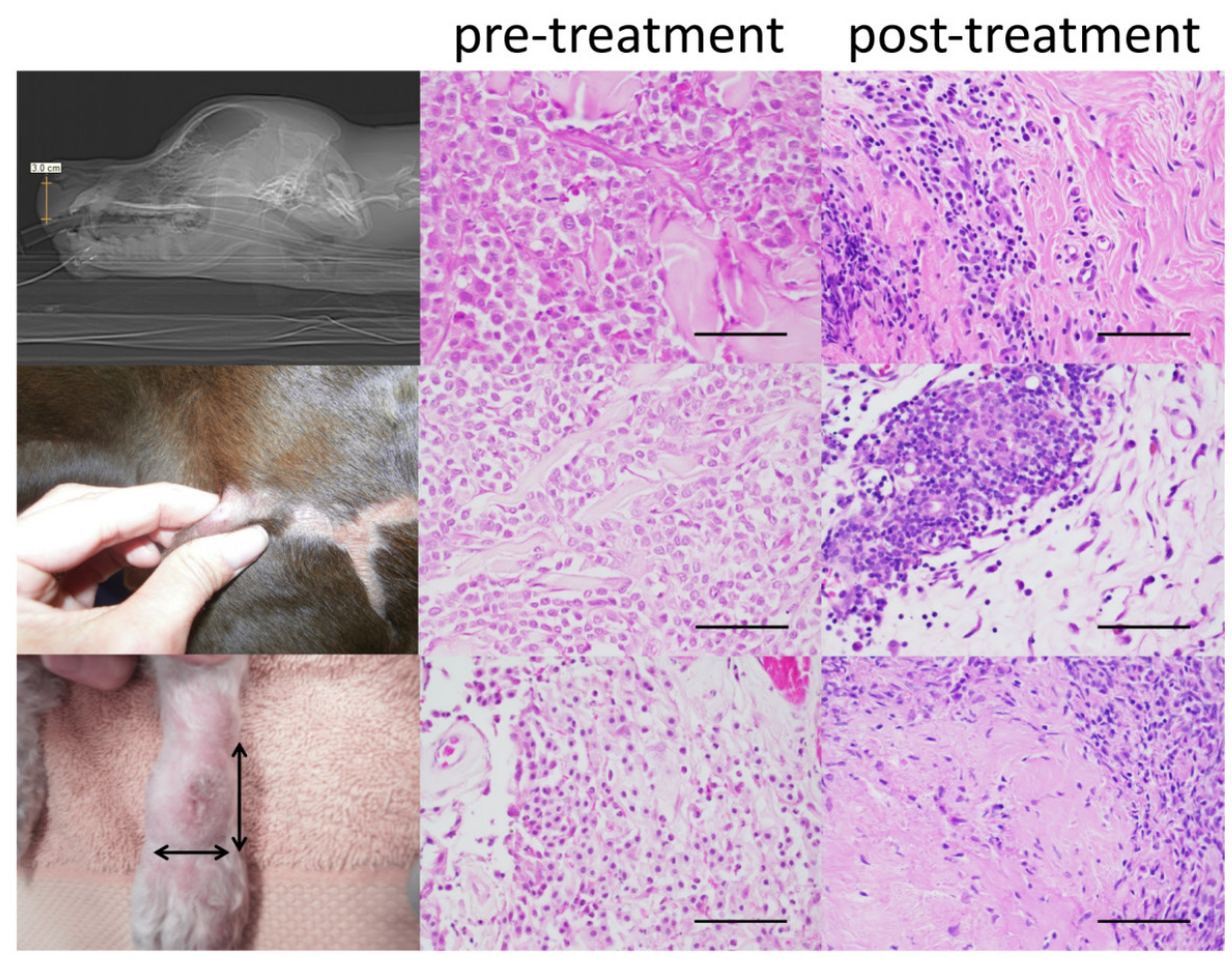

\section{pre-treatment post-treatment}

B

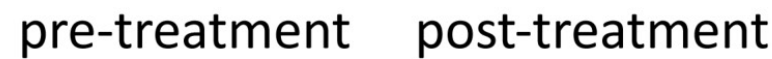

\section{de novo}

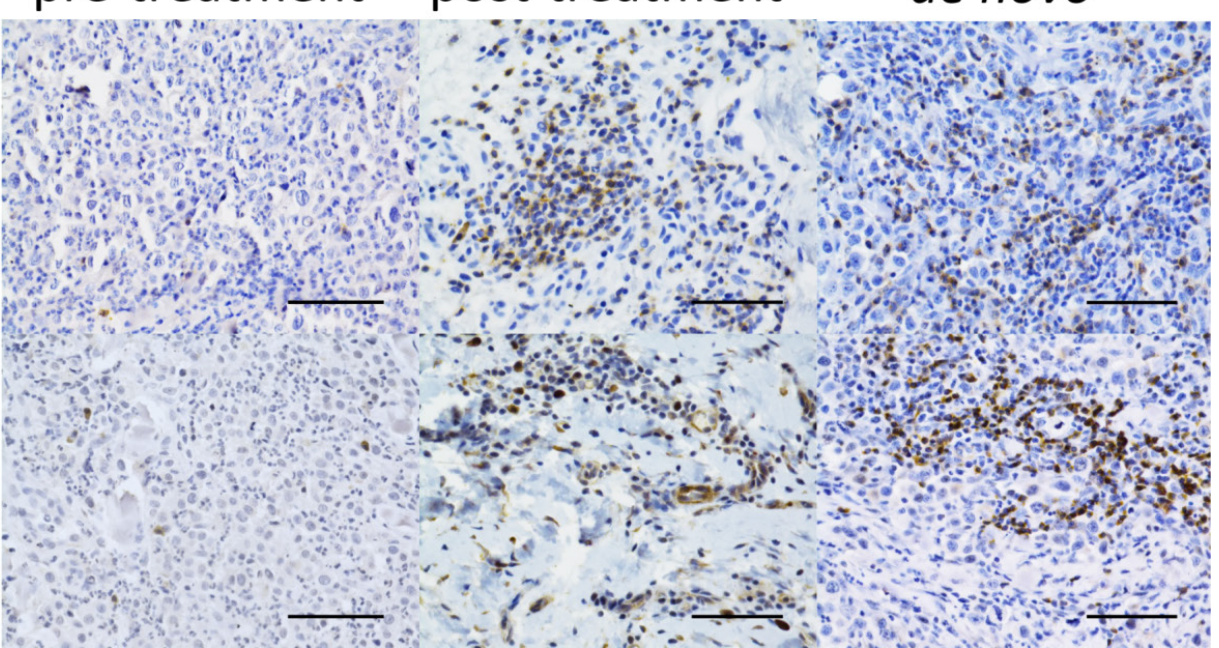

Figure 2 Histologic features of dogs showing regression. (A) Left column: macroscopic images, middle column: histology of pretreatment biopsies, right column: histology of post-treatment biopsies; in all three cases, the tumor has completely regressed and residual lymphocyte, plasma cell, and macrophage infiltrates are present in the dermal connective tissue. H\&E stains magnification 400x, scale bar 50 microns. Top row: pretreatment medical imaging of 'Rottweiler' taken on October 9, 2012, showing a $3 \mathrm{H} \times 2.5 \mathrm{~W} \times 1.8 \mathrm{D} \mathrm{cm}$ tumor within the nasal philtrum and lip (lateral view shown); pretreatment biopsy (September 27,2012 ) showing a high-grade mast cell tumour; post-treatment biopsy of the site of tumor regression (October 23, 2013) approximately 4.5 months postinjection. Middle row: pretreatment morphology of the lateral thigh tumor in 'German shorthaired pointer' on October 22, 2013, large surgical scars from the previous radical surgical resection are visible; pretreatment biopsy (July 11, 2013) showing a low-grade MCT; post-treatment biopsy at the site of tumor regression (November 29, 2013) approximately 22 days post-injection. Bottom row: pretreatment morphology of limb MCT in 'miniature poodle' on February 25, 2015; initial pretreatment biopsy (February 25, 2015) low-grade MCT; post-treatment autopsy tissue (November 13, 2015) at the site of tumor regression, approximately 8.5 months after injection. (B) Immunohistochemistry for T (CD3) top row and B (CD79a) bottom row lymphocytes on paraffin-embedded formalin-fixed tissue of high-grade MCTs from 'Rottweiler'. Left: pretreatment biopsy taken of the nasal/lip tumor (September 27, 2012) shows very rare T cells and rare plasma cells, clusters of lymphocytes and immune cells were absent. Middle: post-treatment biopsy of the site of lip/nasal tumor regression (October 23, 2013) shows clusters of co-associated T and B lymphocytes (serial sections). Right: representative de novo high-grade mastocytoma arising on the neck 17 weeks post-complete Freund's adjuvant treatment for the initial nasal/lip mass. Clusters of T and B lymphocytes were prominent throughout the tumor, which did not reoccur despite incomplete surgical resection. All panels magnification 400x; scale bar 50 microns. Note: vascular wall staining is a feature of the CD79a antibody. 

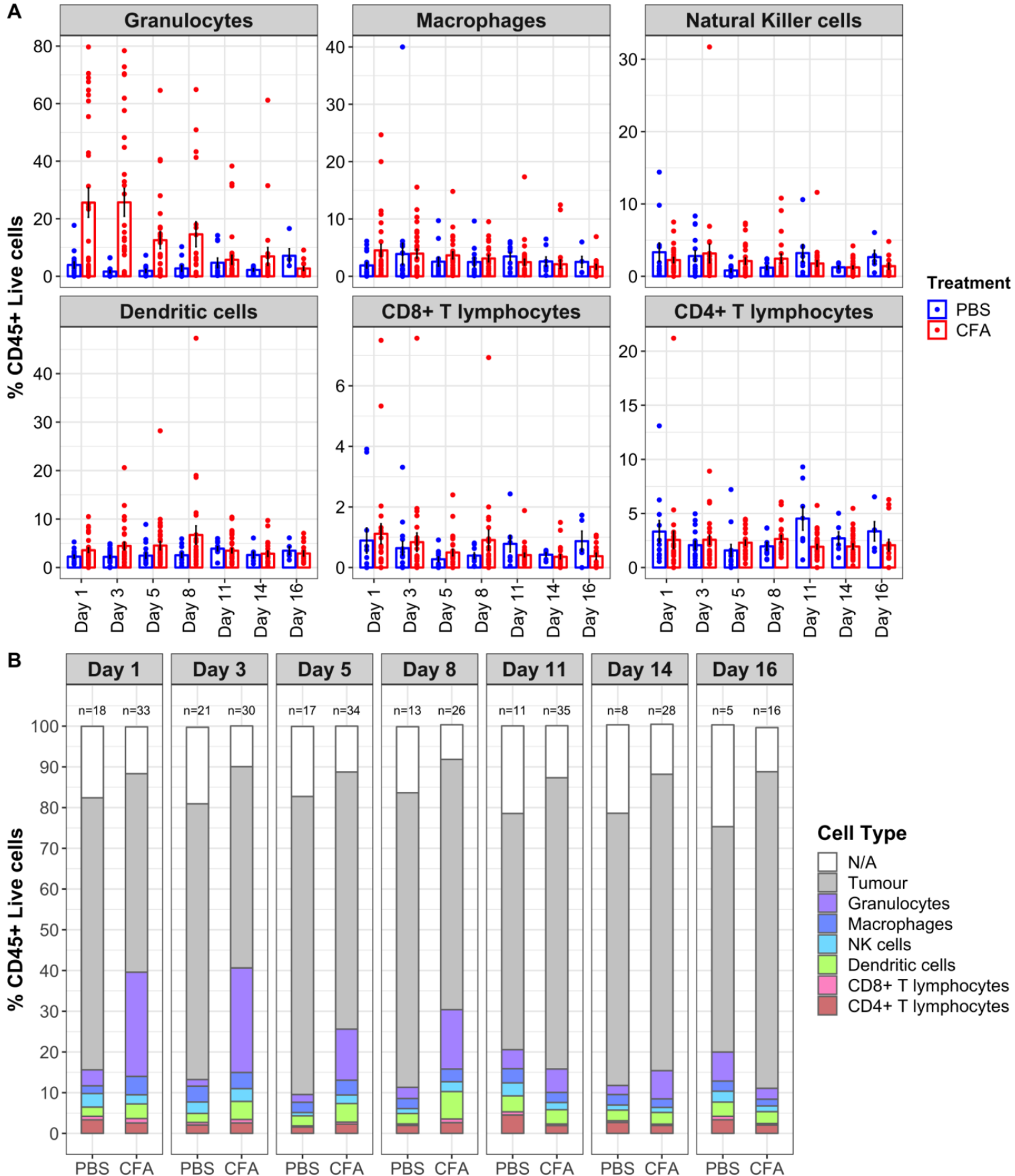

Figure 3 Enumeration of tumor-infiltrating immune cells in P815 tumors following treatment. Tumor-infiltrating immune cells identified by fine-needle aspirates (FNAs) of P815 tumors in mice. Tumor samples were collected by FNA on days 1, 3, 5, 8, 11, 14 , and 16 following CFA/PBS treatment and analyzed by flow cytometry to identify tumor cells and tumor-infiltrating immune cells using the gating strategy previously described (Carroll et al, 2015). (A) The percentage of tumor-infiltrating immune cells are shown for individual CFA-treated mice (red dots) and PBS-control treated mice (blue dots). To avoid fluctuations in percentages due to low numbers of cells, any percentages from populations with less than 15 cells in the previous gate were excluded. The raw means and SEs are shown. A linear mixed effects model (to determine which cell types changed significantly in response to treatment, when considering overall data from all seven sampling days) revealed a significant effect of CFA treatment on the infiltration of granulocytes $(p=0.0025)$ and dendritic cells $(p=0.038)$. However, no significant increases in any of the other cell types were observed: CD4 $4^{+}$cells $(p=0.8691), C D 8^{+} T$ cells $(p=0.2232)$, NK cells $(p=0.468)$, and macrophages $(p=0.6494)$. Contrast analyses were also performed for each FNA day (not corrected for multiple comparisons). CFA-treated mice had significantly increased granulocytes on days $1(p<0.0001), 3(p<0.0001)$, and $5(p=0.0438)$ post-treatment and significantly increased dendritic cells on day 8 post-treatment $(p=0.0037)$. In addition, macrophages were increased on day $1(p=0.0385)$ and CD4 cells decreased on day $11(\mathrm{p}=0.0137)$. Data pooled from six separate experiments. These data were used to generate (B). (B) The relative percentages of tumor-infiltrating immune cells and tumor cells for CFA-treated and PBS-control mice are shown, and the number of mice analyzed at each time point is shown above each bar. CFA, complete Freund's adjuvant; PBS, phosphate-buffered saline. 


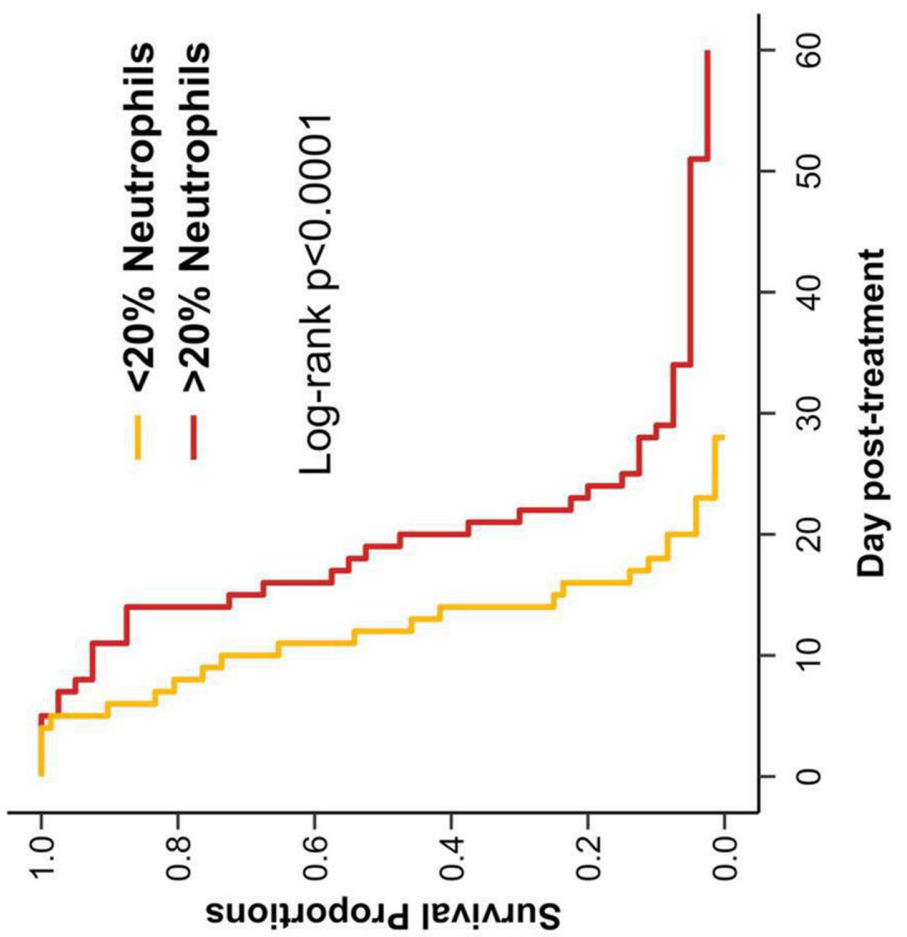

$m$

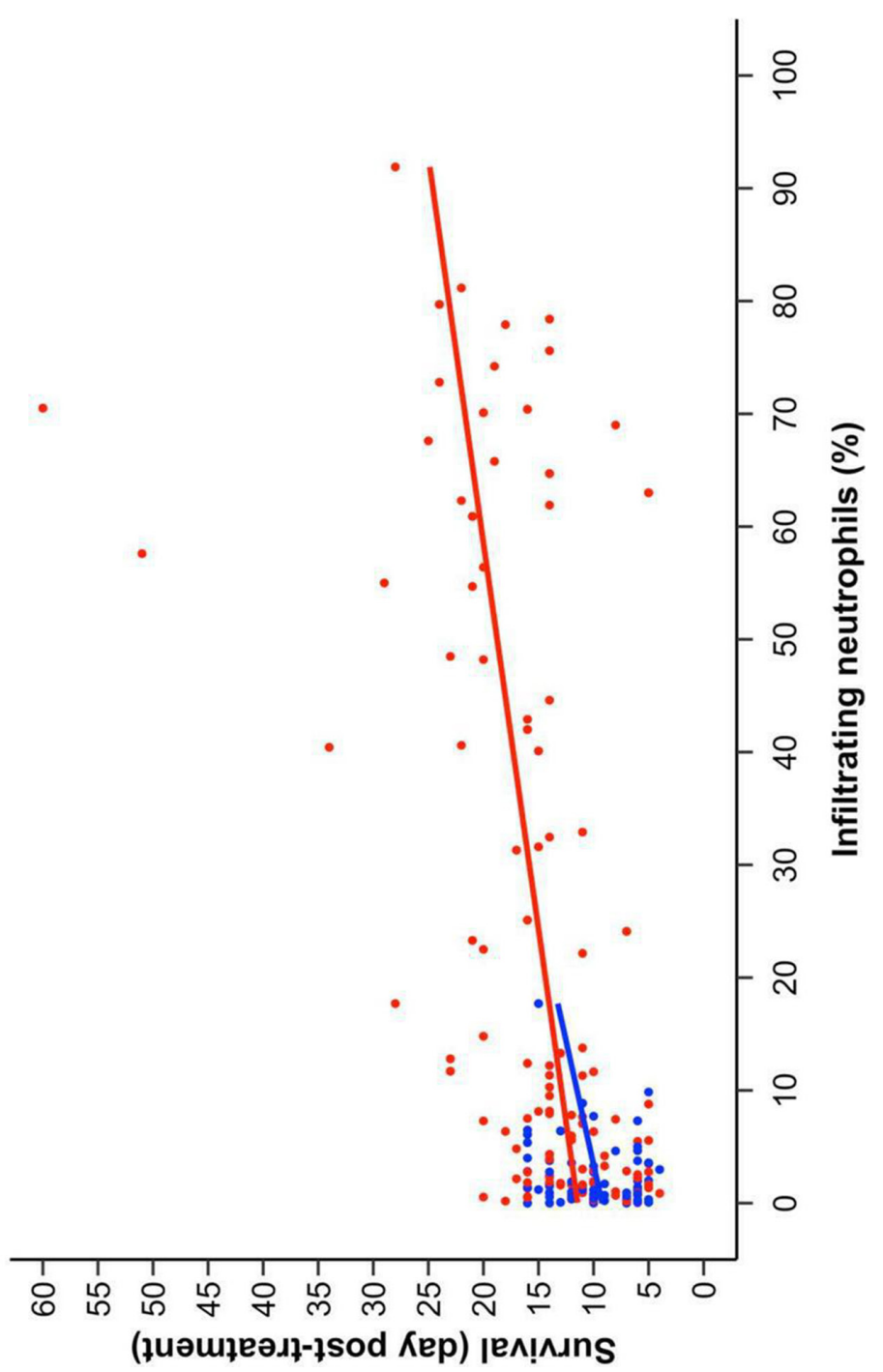

Figure 4 (Continued) 
Figure 4 Early granulocyte infiltrate is the key to treatment success of P815 tumors. (A) The highest percentage of neutrophils identified on days 1-5 for each mouse is plotted against survival time. PBS-treated mice are shown in blue, CFA-treated mice are shown in red. Lines were fitted using a linear model with $\mathrm{R}$ software. Analysis of variance showed that infiltration of neutrophils by day 5 is highly associated with survival $(p<0.0001)$ and that this association is independent of treatment $(p=0.679)$. PBS $n=69$, CFA $n=112$. (B) CFA-treated mice (from A) were stratified based on the highest percentage of tumorinfiltrating neutrophils identified by FNA on days 1 to 5 post-treatment (mice with $<20 \%$ neutrophils $(n=72$, orange) and $>20 \%$ $(n=40$, red)). Survival analyses showed a statistically significant difference in survival between groups (log RANK $p<0.0001)$. The $20 \%$ cut-off was chosen based on the maximum neutrophil infiltrate in PBS-treated mice, as seen in figure 4A. Data pooled from 18 separate experiments, in which FNA analysis was performed at least once on, or before, day 5. CFA, complete Freund's adjuvant; FNA, fine-needle aspirate; PBS, phosphate-buffered saline.

with saline (control). Even in this highly controlled cancer model, there was substantial variation in the proportion of immune infiltrates induced by intratumoral CFA injection (figure 3A). Importantly, mice with the highest levels of granulocytes at days 1-5 post intratumoral CFA injection lived significantly longer than mice with low granulocyte infiltrates (figure 4). Thus, in this model, early immune cell infiltration into the tumor was predictive of treatment benefit. The granulocyte infiltrate was shown to be composed almost entirely of neutrophils. Depletion of neutrophils in the P815 model led to shorter survival and abrogated the efficiency of CFA treatment (figure 5).

\section{Human trials}

Following evidence of benefit of CFA treatment in natural and induced animal models of mastocytoma, breast cancer, and melanoma, the question obviously arises: can this treatment be of benefit to human cancer patients? We provide preliminary reports from two human studies of CFA injected intratumorally in human cancer patients.

Four patients were treated at the Arlesheim Klinik in Switzerland; a 77-year-old man with incurable non-small cell lung cancer (NSCLC), a 17-year-old girl with metastatic osteosarcoma, and two 54-year-old women with invasive ductal breast cancer (one lost to follow-up). These patients received between one and four injections of CFA (in this series of patients, emulsified with Viscum). Details are given in online supplemental table 2A. One breast cancer patient exhibited local control for 2 years, confirmed by repeated ultrasound and MRI scans, but ultimately progressed.

Eight patients were treated as part of an ongoing phase I clinical trial being performed at The Canberra Hospital, Australia (Australian New Zealand Clinical Trials Registry: ACTRN12616001637437). In this ongoing dose-escalation study, two cohorts of previously treated patients with advanced cancer received either $0.5 \mathrm{~mL}$ or 1 $\mathrm{mL}$ of CFA on day 1 (online supplemental table 2B). CFA was administered into primary or metastatic cancer sites either in clinic or under imaging guidance, depending on tumor location. All patients had a mandatory tumor biopsy on day 5 ( 4 days after CFA injection) and were allowed to undergo optional tumor biopsy on day 28 to gauge the level of immune infiltrates.

Five of the first eight patients treated showed evidence of substantial immune infiltrate into the tumor, accompanied by extensive tumor necrosis 4 days after treatment (figure 6A and online supplemental figure 3). Clinical assessments and tumor biopsies did not show significant differences between patients who received $0.5 \mathrm{~mL}$ or $1 \mathrm{~mL}$ CFA. Patients are currently being enrolled into a 2 mL CFA cohort. Treatment was well tolerated in all patients from both centers. Side effects attributed to the therapy were limited to temporary pain at the injection site, inflammation around the injected tumor, and overnight fever; each seen in some patients. Thus, side effects compare very favorably to standard cancer therapies.

One 69-year-old man with metastatic renal cell cancer was treated with CFA after progressing on multiple lines of standard treatments including tyrosine kinase inhibitors. He underwent three doses of $1 \mathrm{~mL}$ CFA ( 6 weeks interval between treatments) that was directly administered into a neck tumor deposit. His serial imaging and histology from tumor biopsy are shown in figure 6 and online supplemental figure 4. Extensive immune infiltrate and tumor necrosis are shown in figure 6A. Figure $6 \mathrm{~B}-\mathrm{D}$ shows CT scans performed before treatment and 6 weeks after the second injection of CFA. In figure 6B, the injected neck tumor deposit can be seen to decrease in size and exhibit radiological changes. Furthermore, a distant lung metastasis was also shown to shrink substantially, accompanied by a decrease in plural effusion (figure 6C). Other metastases, however, were stable or increased in size (figure 6D and online supplemental figure 4).

\section{DISCUSSION}

In this paper, we provide proof of concept that intratumoral injection of CFA emulsified with saline can act as an effective cancer immunotherapy in a proportion of animal models with cancer (both experimentally induced and naturally occurring). We also show that this therapy is safe for use in human cancer patients and may provide benefit to a proportion of patients, thus warranting further clinical trials. Further, we have demonstrated through the use of traditional laboratory animal models, and naturally occurring cancers in two companion animal species (dogs and horses), an alternative comparative oncology methodology for defining proof of concept, efficacy and safety in novel cancer therapies.

Mouse models are excellent for studying the early phases of the antitumor immune response. CFA prolonged life in two mouse cancer models: P815 mastocytoma and 4T1 mammary cancer (figure 1), with complete regressions seen in the former. In the P815 model, improved 

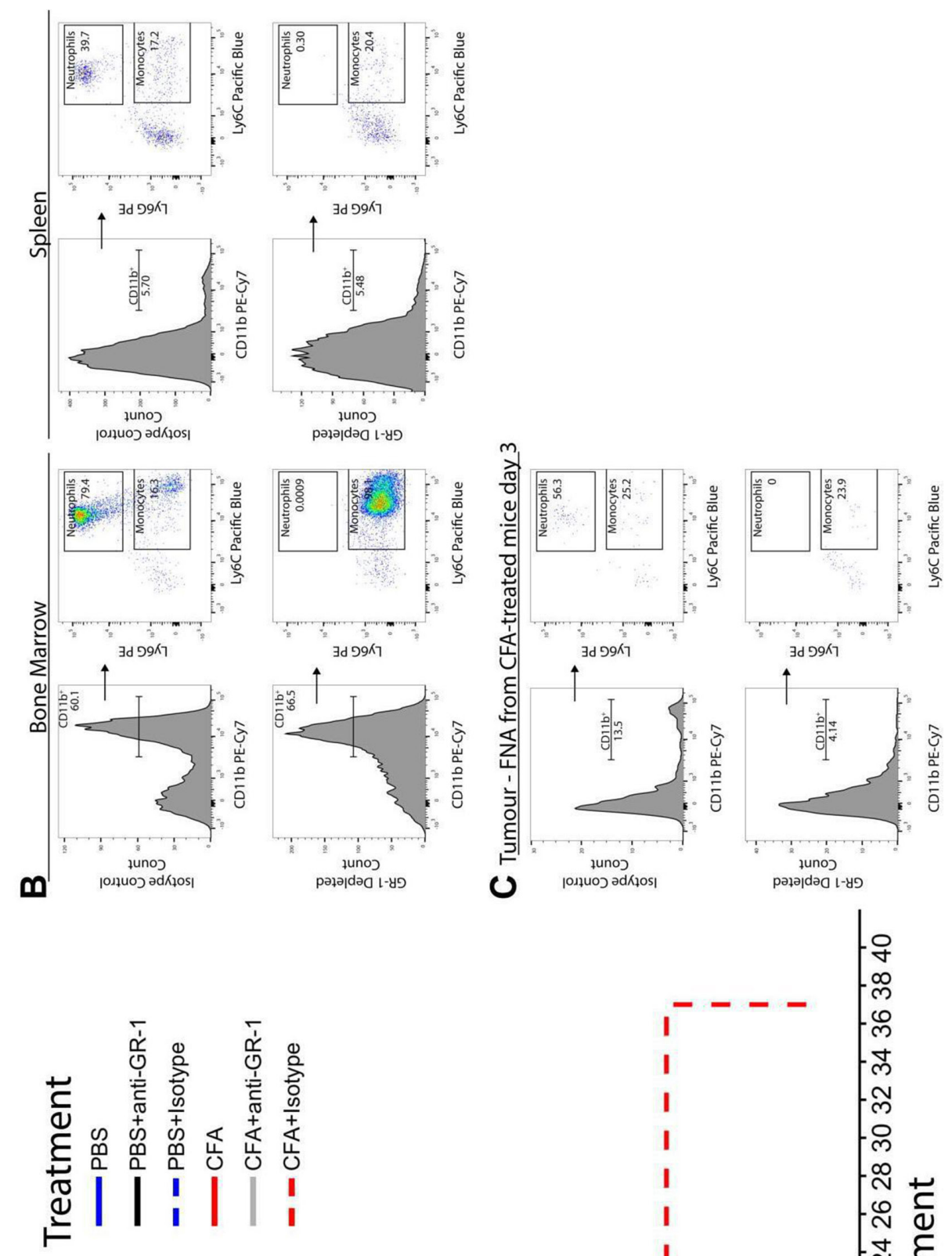

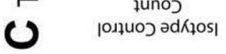

Figure 5 (Continued)

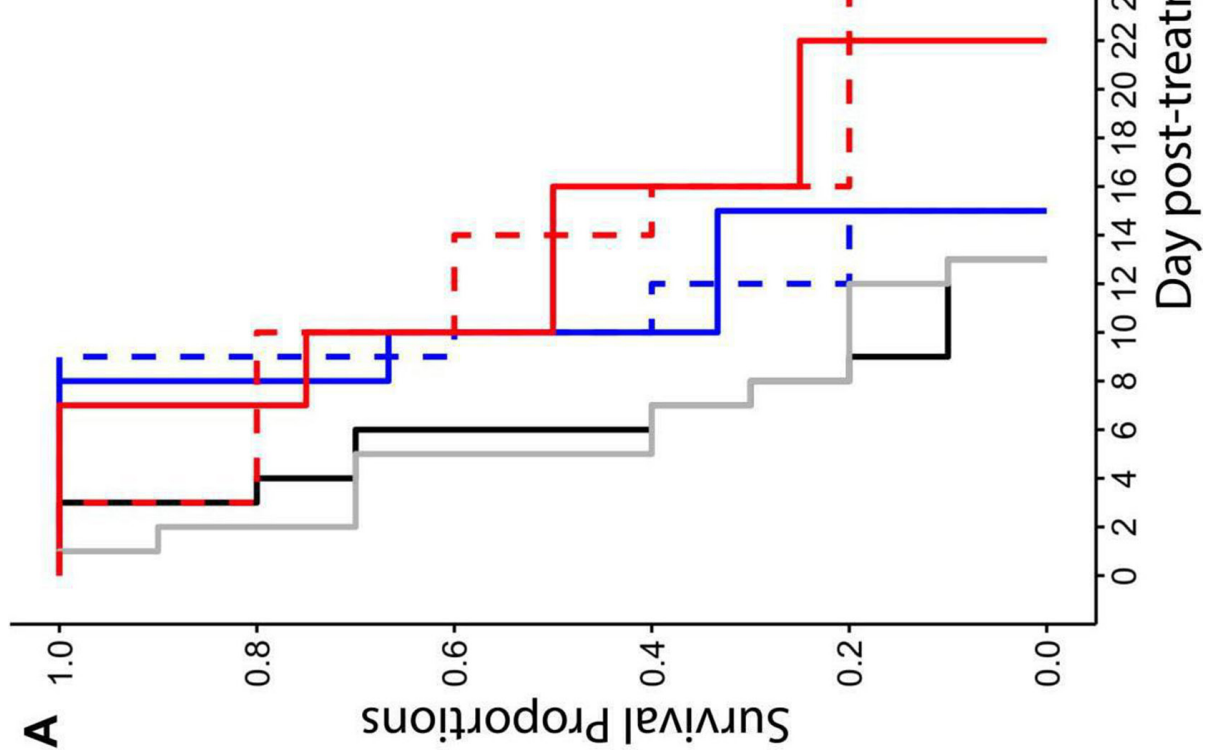


Figure 5 Neutrophil depletion abrogates the survival advantage of CFA treatment. (A) Mice bearing P815 tumors were treated with intratumoral CFA or PBS for controls. Mice additionally received either $0.2 \mathrm{mg}$ anti-GR-1(RB6-8C5) or anti-isotype control antibody (LTF-2) by intraperitoneal injection every 2-3 days, beginning 3 days prior to CFA /PBS treatment. For statistical analysis, mice were stratified by treatment and by neutrophil status (neutrophil depleted or non-depleted). No interaction between neutrophil depletion and treatment was observed $(p=0.230)$, indicating that treatment had no effect on the survival of neutrophil-depleted mice, and neutrophil depletion was found to significantly reduce survival $(p=0.00016$, HRs: nondepleted=1, GR-1-depleted=4.28). The median survival of CFA+anti-GR-1 mice was 5 days post-treatment compared with 13 days and 14 days for CFA and CFA+isotype control mice, respectively. The median survival of PBS+anti-GR-1 mice was 6 days post-treatment compared with 10 days for both PBS and PBS+isotype control mice. CFA $n=4$, PBS $n=3, C F A+$ anti-isotype $n=5$, PBS+anti-isotype $n=5$, CFA+anti-GR-1 $n=10$, PBS+anti-GR-1 $n=10$. Data pooled from two separate experiments. (B) Assessment of neutrophil depletion in the spleen and bone marrow. Bone marrow cells from a non-depleted control mouse and a GR-1-depleted mouse were processed and stained with 7-AAD, anti-CD45.2-Alexa fluor 488, anti-CD11b-PE-Cy7, anti-Ly6GPE, and anti-Ly6C-Brilliant violet 421 (BV421). Cells have been gated for CD45.2 positive, live (7-AAD negative) singlet cells. The histograms show that the proportion of myeloid cells $(C D 11 b+)$ were similar in both non-depleted control mice (top panels) and GR-1-depleted mice (bottom panels). From the myeloid population, distinct populations of both neutrophils (Ly6G+Ly6C+) and monocytes (Ly6G- Ly6C+) could be identified in the non-depleted control mice. However, the neutrophil population is absent from the GR-1-depleted mouse, indicating that neutrophils had been successfully depleted using the anti-GR-1 antibody (RB68C5) (C). Assessment of neutrophil depletion in mice with P815 tumors. neutrophils were depleted from mice bearing P815 tumors starting 3 days prior to CFA treatment using $0.2 \mathrm{mg}$ anti-GR-1 antibody (RB6-8C5), which was re-administered every 2-3 days. FNAs were taken from P815 tumors 3 days post-CFA treatment to ensure successful depletion of neutrophils by flow cytometry. FNA samples were pooled into groups from GR-1-depleted and isotype control mice. The neutrophil population $(\mathrm{CD} 11 \mathrm{~b}+\mathrm{Ly} 6 \mathrm{G}+\mathrm{Ly} 6 \mathrm{C}+)$ is present in the isotype control mice. CFA, complete Freund's adjuvant; FNA, fine-needle aspirate; PBS, phosphate-buffered saline; 7-AAD, 7-aminoactinomycin-D.

survival was dependent on early neutrophil infiltration into the tumor. Dogs allow longer term investigation in naturally occurring cancers, which are genetically and behaviourally similar to human cancers, and have developed in co-evolution with the immune system. ${ }^{31}$ Our canine studies demonstrate the effectiveness of intratumoral CFA treatment in naturally occurring canine mastocytomas of both high and low grade, with a $21 \%$ complete response rate and a $35 \%$ overall response rate. It should be noted that we are targeting dogs with advanced terminal malignancies in our trial, and some animals have succumbed to their cancer in less than 2-4 weeks we predict it takes to mount a significant antitumor immune response. Histology of dog tissues after tumor regression (figure 2) showed B and T cell infiltration at the site of tumor regression, and in a de novo (not injected) cancer, providing evidence of a systemic immune response to the treatment in addition to regression of the injected tumor. In the dog trial, regressions were demonstrated after failure of chemotherapy and radical surgery, as well as in an untreated lesion, thus intratumoral CFA can be considered both as a first-line treatment, or after failure of other therapies.

Together, our data suggest that early stimulation of an innate immune response is the key to treatment success, sufficient for the resolution of some injected tumors. The ensuing adaptive ( $\mathrm{T}$ and $\mathrm{B}$ cell) immune response (seen in late dog histology) is likely to be the key to the control of any metastases. This is very much in agreement with a similar hypothesis put forward by Colombo and colleagues. ${ }^{32}$

In our human patients, we show that intratumoral injection of CFA is safe and well-tolerated. Histology from tumor biopsies taken 4 days after CFA injection is consistent with our mouse flow cytometry data, with inflammatory immune cells seen in $5 / 8$ patients, in each case accompanied by extensive tumor necrosis. We cannot expect small phase I trials in mostly end-stage cancer patients to conclusively demonstrate treatment efficacy. Nonetheless, at least one patient (patient 4 in the Canberra trial, figure 4) is likely to have derived benefit from the therapy.

The use of bacteria in cancer treatment has a long history, ${ }^{33}$ but also a far more recent one. Live BCG is the current standard treatment for superficial bladder cancer $^{34}$ (note that CFA, used in our study, contains a closely related dead mycobacterium). Two seminal papers in 2012 showed that common chemotherapies also act through bacteria: pretreatment with antibiotics in mouse models abrogated the effectiveness of chemotherapy. ${ }^{11} 12$ Chemotherapy was shown to damage the intestinal lining, allowing translocation of bacteria across the gut wall with bacteria found in the mesenteric lymph nodes and spleen. ${ }^{11}$ Three classes of cancer therapeutic checkpoint inhibitors, anti-PDL-1, anti-CTLA4, and anti-PD-1, have shown similar dependence on gut bacteria, ${ }^{1435} 36$ with the effectiveness of the latter two also impaired by antibiotics. Routy et $\mathrm{al}^{14}$ note the presence of bacterial-induced granulomas in the cancer after treatment of mice with anti PD-1. All three checkpoint inhibitors are known to cause gastrointestinal toxicities in patients and so impair the integrity of the intestinal lining. Associations between enterocolitis or immune-related adverse events after checkpoint inhibitor therapy and improved patient outcomes have been noted (reviewed by Soularue et $a \hat{l}^{37}$ ). Importantly, retrospective studies of patients taking checkpoint inhibitors have shown that co-prescription of antibiotics leads to significantly shorter survival. ${ }^{14} 38$ Taken together, these data support the thesis that the success of many cancer therapies relies on escape of bacteria from the gut; these bacteria can end up in tumors and in tumor-draining lymph nodes where they are able to activate first the 

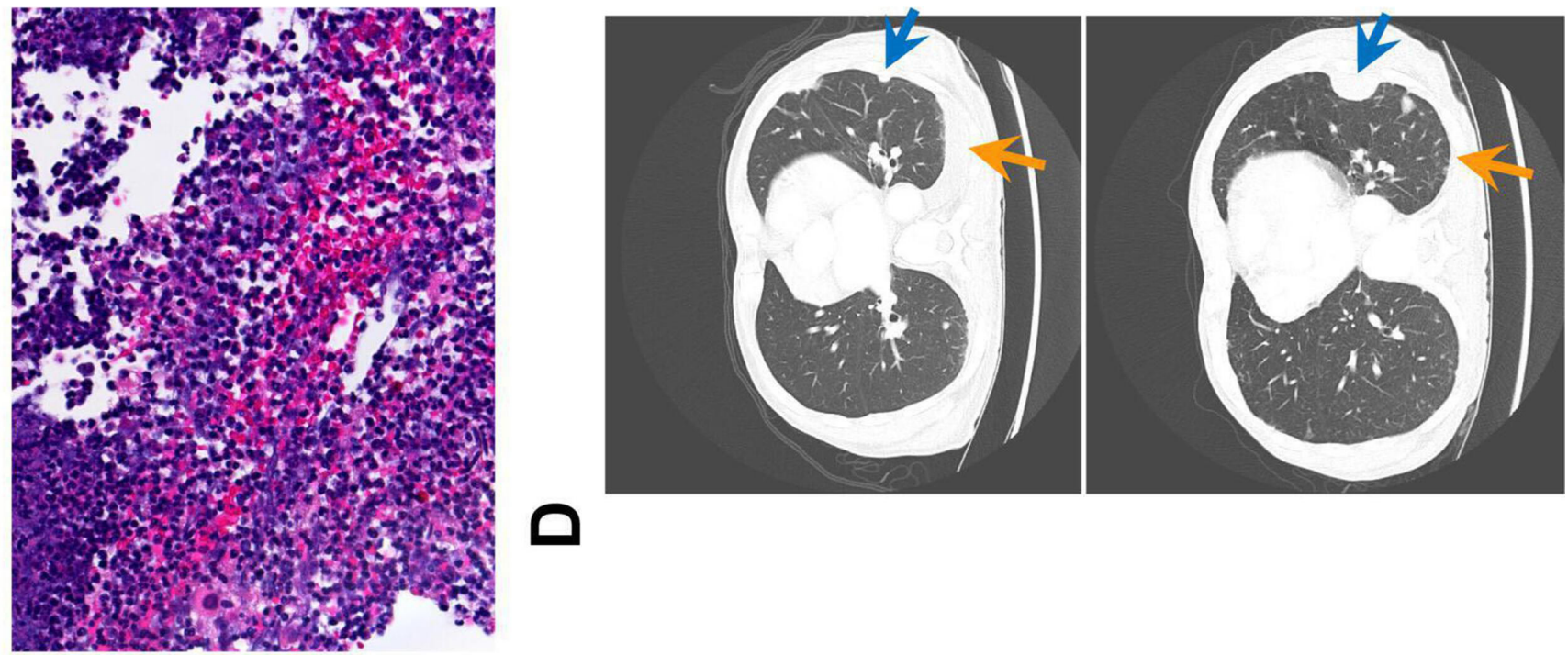

○
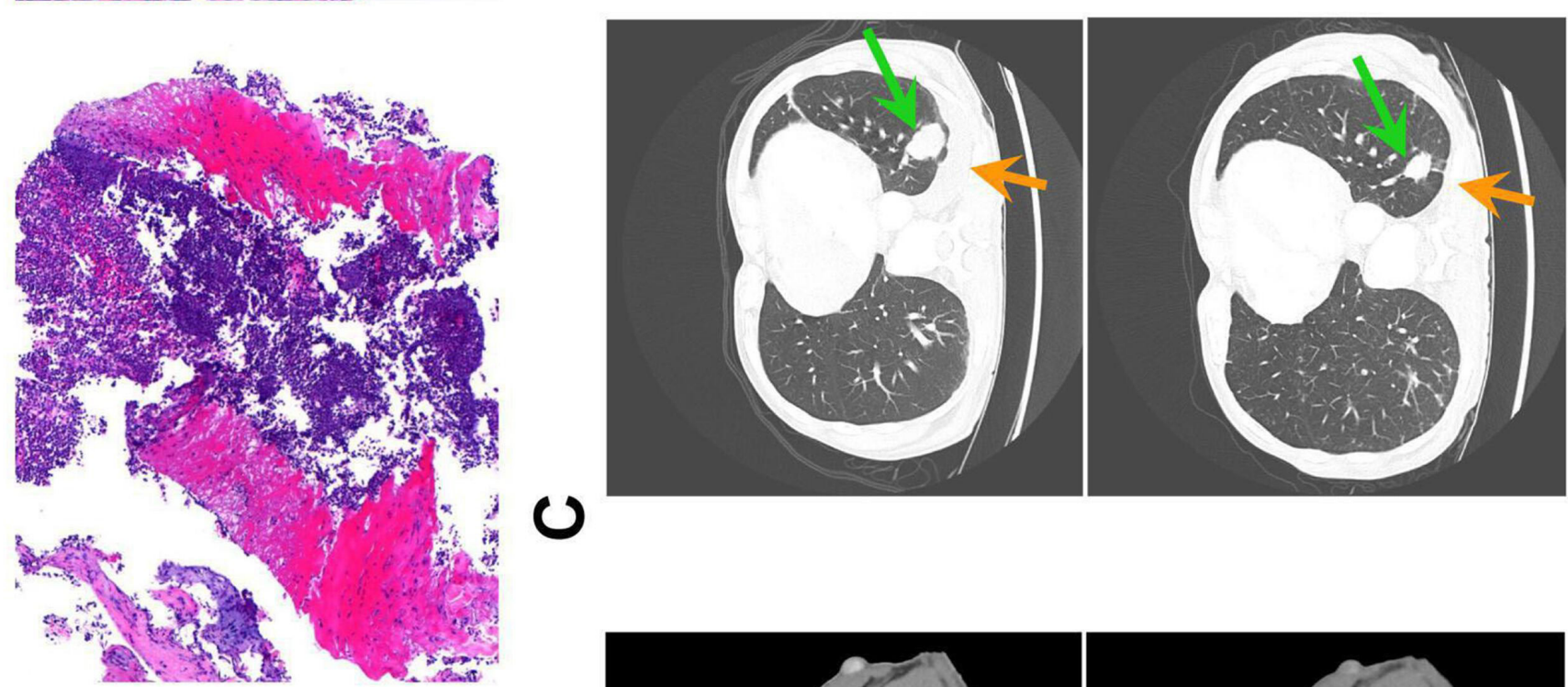

0
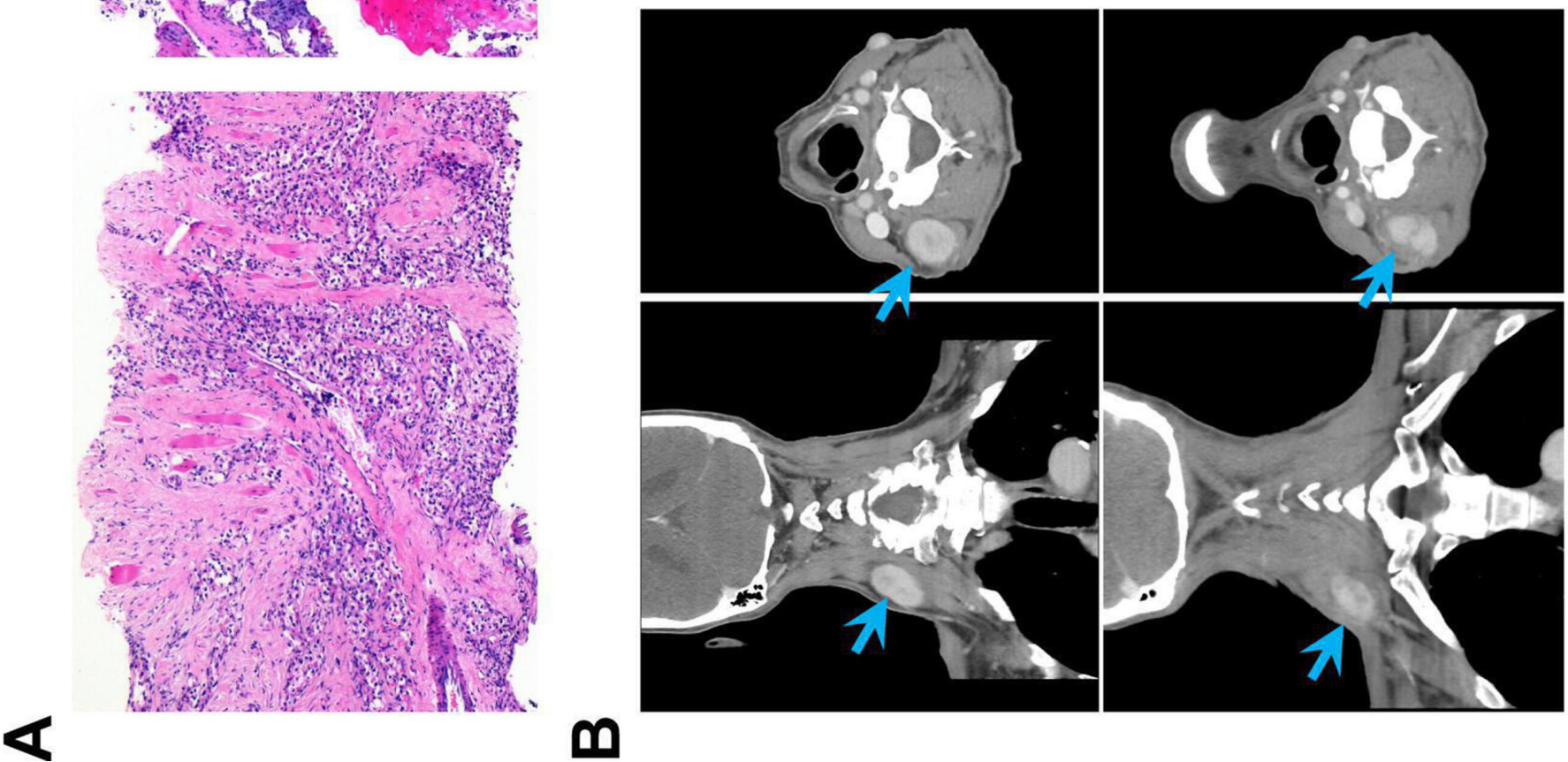

Figure 6 Histology and CT scans of treated patient. Patient 4 received three injections at 6 -week intervals of $1 \mathrm{~mL}$ of CFA into a neck deposit of clear cell carcinoma of the kidney. (A) H\&E sections of tumor on initial biopsy taken 1 year before CFA Figure 6 (Continued) 
treatment (left $\times 100$ ) and on biopsy taken 4 days after the first injection of CFA (middle $x 100$, right $\times 400)$. The post-treatment biopsy shows extensive immune infiltrate (predominantly neutrophils and macrophages) and no viable tumor. (B-D) CT scans taken 8 days before the first injection (top row) and 5 weeks after the second injection (bottom row). An earlier scan is shown in online supplemental figure 5. (B) Scans of the neck showing the injected lesion (light blue arrow; $40 \times 27 \mathrm{~mm}$ before treatment, $28 \times 18 \mathrm{~mm}$ after two injections). (C) Scans of lung showing tumor shrinkage (green arrow; $33 \mathrm{~mm}$ before treatment, $21 \mathrm{~mm}$ after) and substantially reduced pleural effusion (orange arrows). (D) Scans of lung showing a tumor that continued to grow despite treatment (dark blue arrow; 9 mm before treatment, 33 mm after). CFA, complete Freund's adjuvant.

innate immune response and then to activate or re-activate the anticancer acquired immune response. ${ }^{17}$

Evidently, if this is correct, a bacterial-based intratumoral cancer treatment could synergize with many current cancer treatment options or be used alone with dramatically fewer side effects. In support of this is (1) the original work of Coley, ${ }^{33}$ (2) our current study, and (3) the recent paper by Sagiv-Barfi et al, which uses intratumoral injection of TLR9 or of TLR7/8 agonists (mimicking bacterial or viral PAMPs, respectively ${ }^{2}$; these are effective at eliminating tumors into which they have been injected, and when combined with an immune-stimulating antibody against OX-40, produce a spectacular, systemic curative immune response in mice.

The question of which bacteria are most effective in cancer therapy has been addressed recently by a series of studies looking at gut bacteria in patients and animals who do or do not respond to chemotherapy or checkpoint inhibitor therapy. Different papers reach largely different conclusions. This suggests that many different bacterial genera may be able to effectively stimulate the anticancer immune response. Anticancer effects of different viral and bacterial vectors being trialed for oncolytic therapy are also likely to be based, at least in part, on the same mechanism, further increasing the range of potentially effective microbiological agents.

Our study investigated injection of emulsified CFA (a slow-release preparation of killed mycobacteria) directly into tumors. Experiments are underway to further elucidate the mechanism of action and to see if the efficacy can be improved. Given the low side effects, potentially wide applicability, and low cost of use (making this therapy applicable even in underdeveloped countries), our therapy warrants larger-scale investigation.

\author{
Author affiliations \\ ${ }^{1}$ Research School of Biology, Australian National University, Canberra, Australian \\ Capital Territory, Australia \\ ${ }^{2}$ Department of Medical Oncology, Canberra Hospital, Canberra, Australian Capital \\ Territory, Australia \\ ${ }^{3}$ Medical School, Australian National University, Canberra, Australian Capital \\ Territory, Australia \\ ${ }^{4}$ School of Veterinary Science, The University of Queensland, Gatton, Queensland, \\ Australia \\ ${ }^{5}$ Adelaide Plains Equine Clinic, Gawler, South Australia, Australia \\ ${ }^{6}$ Klinik Arlesheim, Haus Wegman, Arlseheim, Switzerland \\ ${ }^{7}$ ACT Pathology, Canberra Hospital, Canberra, Australian Capital Territory, Australia \\ ${ }^{8}$ Canberra Imaging Group, Canberra, Australian Capital Territory, Australia \\ ${ }^{9}$ Biological Data Science Institute, Australian National University, Canberra, \\ Australian Capital Territory, Australia
}

Twitter Andrew A Almonte @andrew_almo
Acknowledgements We would especially like to thank all patients with cancer who volunteered for treatment and their families. Thank you to Olive Doig, Clinical Trials Manager, and to Drs Robert Allen and Sayed Ali. AF would like to thank Claude Fahrer for helpful discussions, Roger Kelly and Hannah Clarke for facilitating this study. RA would like to thank Chiara Palmieri, Greg Simmons, Hanna Long, Andrea Schaffer-White. Thank you to the owners and referring veterinarians of the pet dogs.

Contributors CSEC and ERA performed mouse experiments, analyzed data, and edited manuscript. AAA analyzed mouse experiments and edited manuscript. LM organized Canberra clinical trial, drafted human ethics, and recruited patients. KFE, $\mathrm{MB}$, and $\mathrm{CL}$ are veterinarians who designed and executed the canine clinical trial including patient treatment, data, sample collection, and analysis. PMP assisted with the research trial and conducted the histology and immunohistochemistry on the dog and horse samples. JM and EWH are veterinarians who performed equine trial, including provision and analysis of equine ultrasounds. K-MS treated patients in Canberra trial and provided input into experimental planning and analysis. RT treated patients in Canberra trial who required injection under ultrasound or CT; assessed and analyzed radiology. JED organised and interpreted Canberra patient histology samples. TN provided expert statistical advice for design and interpretation of mouse experiments. AH and M0 treated Swiss patients. DY was in charge of Canberra Hospital clinical trial and edited manuscript. RA was in charge of the dog clinical trial, interpretation of samples collected for histology and immunohistochemistry for dogs and horses, manuscript drafting, and editing. AF oversaw study; was in charge of mouse experiments and analysis of data; prepared CFA and helped draft human ethics for Canberra clinical trial; helped prepare figures and tables; and wrote manuscript.

Funding Canberra trial: Canberra Hospital Private Practice Fund, Janice and Ron Parker Fund. Mouse preclinical studies: Lea Chapuis Memorial Fund. Canine preclinical studies: The John and Mary Kibble Trust (grants CT22492, CT21335) and the William Peter Richards Bequest for research into veterinary pathology. CSEC, ERA, and AAA was supported by Australian Government Research Training Program Stipend Scholarships. CSEC and K-MS were supported by Max Lindemann Memorial Foundation, Miami.

Competing interests None declared.

Patient consent for publication Not required.

Ethics approval Mouse experiments were approved by the Australian National University (ANU) animal experimentation ethics committee; approval numbers A2011/020, A2014/03, A2017_46.

Provenance and peer review Not commissioned; externally peer reviewed.

Data availability statement Data are available upon reasonable request. All data relevant to the study are included in the article or uploaded as supplementary information. We are happy to provide any further information. Please contact senior authors DY, MO, RA or AMF.

Supplemental material This content has been supplied by the author(s). It has not been vetted by BMJ Publishing Group Limited (BMJ) and may not have been peer-reviewed. Any opinions or recommendations discussed are solely those of the author(s) and are not endorsed by BMJ. BMJ disclaims all liability and responsibility arising from any reliance placed on the content. Where the content includes any translated material, BMJ does not warrant the accuracy and reliability of the translations (including but not limited to local regulations, clinical guidelines, terminology, drug names and drug dosages), and is not responsible for any error and/or omissions arising from translation and adaptation or otherwise.

Open access This is an open access article distributed in accordance with the Creative Commons Attribution Non Commercial (CC BY-NC 4.0) license, which permits others to distribute, remix, adapt, build upon this work non-commercially, and license their derivative works on different terms, provided the original work is properly cited, appropriate credit is given, any changes made indicated, and the use is non-commercial. See http://creativecommons.org/licenses/by-nc/4.0/. 


\section{ORCID iD}

Aude M Fahrer http://orcid.org/0000-0001-8556-0223

\section{REFERENCES}

1 Fahrer AM. A proposal for a simple and inexpensive therapeutic cancer vaccine. Immunol Cell Biol 2012;90:310-3.

2 Sagiv-Barfi I, Czerwinski DK, Levy S, et al. Eradication of spontaneous malignancy by local immunotherapy. Sci Trans/ Med 2018;10. doi:10.1126/scitransImed.aan4488. [Epub ahead of print: 31012018 .

3 Nauts HC, Swift WE, Coley BL. The treatment of malignant tumors by bacterial toxins as developed by the late William B. Coley, M.D., reviewed in the light of modern research. Cancer Res 1946;6:205-16.

4 Nauts HC, McLaren JR. Coley toxins-the first century. Adv Exp Med Biol 1990;267:483-500.

5 Sedighi M, Zahedi Bialvaei A, Hamblin MR, et al. Therapeutic bacteria to combat cancer; current advances, challenges, and opportunities. Cancer Med 2019;8:3167-81.

6 Zhou S, Gravekamp C, Bermudes D, et al. Tumour-targeting bacteria engineered to fight cancer. Nat Rev Cancer 2018;18:727-43.

7 Twumasi-Boateng K, Pettigrew JL, Kwok YYE, et al. Oncolytic viruses as engineering platforms for combination immunotherapy. Nat Rev Cancer 2018;18:419-32.

8 Hernández-Luna MA, Luria-Pérez R. Cancer Immunotherapy: priming the Host Immune Response with Live Attenuated Salmonella enterica. J Immunol Res 2018;2018:1-15.

9 Lee S-Y, Yang S-B, Choi Y-M, et al. Heat-Killed Mycobacterium paragordonae therapy exerts an anti-cancer immune response via enhanced immune cell mediated oncolytic activity in xenograft mice model. Cancer Lett 2020;472:142-50.

10 Mathé G, Florentin I, Bruley-Rosset M, et al. Heat-Killed Pseudomonas aeruginosa as a systemic adjuvant in cancer immunotherapy. Biomedicine 1977;27:368-73.

11 Viaud S, Saccheri F, Mignot G, et al. The intestinal microbiota modulates the anticancer immune effects of cyclophosphamide. Science 2013;342:971-6.

12 lida N, Dzutsev A, Stewart CA, et al. Commensal bacteria control cancer response to therapy by modulating the tumor microenvironment. Science 2013;342:967-70.

13 Yu T, Guo F, Yu Y, et al. Fusobacterium nucleatum promotes chemoresistance to colorectal cancer by modulating autophagy. Cell 2017;170:548-63.

14 Routy B, Le Chatelier E, Derosa L, et al. Gut microbiome influences efficacy of PD-1-based immunotherapy against epithelial tumors. Science 2018;359:91-7

15 Baruch EN, Youngster I, Ben-Betzalel G, et al. Fecal microbiota transplant promotes response in immunotherapy-refractory melanoma patients. Science 2021;371:602-9.

16 Matson V, Fessler J, Bao R, et al. The commensal microbiome is associated with anti-PD-1 efficacy in metastatic melanoma patients. Science 2018;359:104-8.

17 Almonte AA, Rangarajan H, Yip D, et al. How does the gut microbiome influence immune checkpoint blockade therapy? Immunol Cell Biol 2021:99:361-72.

18 Marchesi JR, Dutilh BE, Hall N, et al. Towards the human colorectal cancer microbiome. PLoS One 2011;6:e20447.

19 Geller LT, Barzily-Rokni M, Danino T, et al. Potential role of intratumor bacteria in mediating tumor resistance to the chemotherapeutic drug gemcitabine. Science 2017;357:1156-60.
20 Nejman D, Livyatan I, Fuks G, et al. The human tumor microbiome is composed of tumor type-specific intracellular bacteria. Science 2020;368:973-80.

21 Kalaora S, Nagler A, Nejman D, et al. Identification of bacteriaderived HLA-bound peptides in melanoma. Nature 2021;592:138-43.

22 Freund J. The mode of action of immunologic adjuvants. Bib/ Tuberc 1956; $7: 130-48$

23 Carroll CSE, Altin JG, Neeman T, et al. Repeated fine-needle aspiration of solid tumours in mice allows the identification of multiple infiltrating immune cell types. J Immunol Methods 2015;425:102-7.

24 Kiupel M, Webster JD, Bailey KL, et al. Proposal of a 2-tier histologic grading system for canine cutaneous mast cell tumors to more accurately predict biological behavior. Vet Pathol 2011;48:147-55.

25 Gordon I, Paoloni M, Mazcko C, et al. The comparative oncology trials Consortium: using spontaneously occurring cancers in dogs to inform the cancer drug development pathway. PLoS Med 2009;6:e1000161.

26 Paoloni M, Khanna C. Translation of new cancer treatments from PET dogs to humans. Nat Rev Cancer 2008;8:147-56.

27 Roberts NJ, Zhang L, Janku F, et al. Intratumoral injection of Clostridium novyi-NT spores induces antitumor responses. Sci Trans/ Med 2014;6:249ra111.

28 Veterinary cooperative oncology group. Common terminology criteria for adverse events (VCOG-CTCAE) following chemotherapy or biological antineoplastic therapy in dogs and cats v1.1. Vet Comp Oncol 2011;14:417-46.

29 Rosengren Pielberg G, Golovko A, Sundström E, et al. A cisacting regulatory mutation causes premature hair graying and susceptibility to melanoma in the horse. Nat Genet 2008:40:1004-9.

30 Curik I, Druml T, Seltenhammer M, et al. Complex inheritance of melanoma and pigmentation of coat and skin in grey horses. PLoS Genet 2013;9:e1003248.

31 Park JS, Withers SS, Modiano JF, et al. Canine cancer immunotherapy studies: linking mouse and human. J Immunother Cancer 2016;4:97

32 Guiducci C, Vicari AP, Sangaletti S, et al. Redirecting in vivo elicited tumor infiltrating macrophages and dendritic cells towards tumor rejection. Cancer Res 2005;65:3437-46.

33 Coley WB. The treatment of inoperable sarcoma by bacterial toxins (the mixed toxins of the Streptococcus erysipelas and the Bacillus prodigiosus). Proc $R$ Soc Med 1910;3:1-48.

34 Redelman-Sidi G, Glickman MS, Bochner BH. The mechanism of action of BCG therapy for bladder cancer--a current perspective. Nat Rev Urol 2014;11:153-62.

35 Sivan A, Corrales L, Hubert N, et al. Commensal Bifidobacterium promotes antitumor immunity and facilitates anti-PD-L1 efficacy. Science 2015;350:1084-9.

36 Vétizou M, Pitt JM, Daillère R, et al. Anticancer immunotherapy by CTLA-4 blockade relies on the gut microbiota. Science 2015;350:1079-84.

37 Soularue E, Lepage P, Colombel JF, et al. Enterocolitis due to immune checkpoint inhibitors: a systematic review. Gut 2018;67:2056-67.

38 Derosa L, Hellmann MD, Spaziano M, et al. Negative association of antibiotics on clinical activity of immune checkpoint inhibitors in patients with advanced renal cell and non-small-cell lung cancer. Ann Oncol 2018;29:1437-44. 